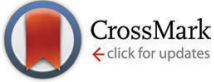

Cite this: Phys. Chem. Chem. Phys., 2015, 17, 6858

Received 3rd December 2014 Accepted 2nd February 2015

DOI: $10.1039 / c 4 c p 05618 c$

www.rsc.org/pccp

\title{
Charge separation and isolation in strong water droplet impacts $\dagger$
}

\author{
F. Wiederschein, ${ }^{a}$ E. Vöhringer-Martinez, ${ }^{* b}$ A. Beinsen, ${ }^{c}$ F. Postberg, ${ }^{\text {de }}$ J. Schmidt, ${ }^{f}$ \\ R. Srama, ${ }^{e}$ F. Stolz, ${ }^{c g}$ H. Grubmüller ${ }^{\mathrm{a}}$ and B. Abel ${ }^{\mathrm{cg}}$
}

\begin{abstract}
Charge separation in condensed matter after strong impacts is a general and intriguing phenomenon in nature, which is often identified and described but not necessarily well understood in terms of a quantitative mechanistic picture. Here we show that charge separation naturally occurs if water droplets/ clusters or ice particles with embedded charge carriers, e.g., ions, encounter a high energy impact with subsequent dispersion - even if the involved kinetic energy is significantly below the molecular ionization energy. We find that for low charge carrier concentrations $\left(c<0.01 \mathrm{~mol} \mathrm{~L}^{-1}\right)$ a simple statistical Poisson model describes the charge distribution in the resulting molecular "fragments" or aggregates. At higher concentrations Coulomb interactions between the charge carriers become relevant, which we describe by a Monte Carlo approach. Our models are compared to experimental data for strong (laser) impacts on liquid micro beams and discussed for the charge generation in cluster-impact mass spectrometry on cosmic dust detectors where particle kinetic energies are below the plasma threshold. Taken together, a simple and intuitive but quantitative microscopic model is obtained, which may contribute to the understanding of a larger range of phenomena related to charge generation and separation in nature.
\end{abstract}

\section{Introduction}

Charge separation and isolation in finite molecular aggregates, droplets, and particles - in particular for water - plays an important role in many areas of science ${ }^{1-6}$ and remains elusive up to the present. Already Lenard noted - more than a century ago - the electrical effects associated with drop breakup near waterfalls. ${ }^{3}$ Blanchard observed that bubble bursting over the oceans releases positively charged jet droplets. ${ }^{7}$ A number of more or less speculative mechanisms have been proposed to account for the observed charges on cloud particles, which ultimately lead to thunderstorms and lightnings. ${ }^{8,9}$ The electrokinetic effect in small micro jets has recently been employed for power generation. ${ }^{10}$ However, most of the charging mechanisms

\footnotetext{
${ }^{a}$ MPI für biophysikalische Chemie, Am Fassberg 11, 37077 Göttingen, Germany

${ }^{b}$ Departamento de Físico-Quimica, Facultad de Ciencias Quimicas,

Universidad de Concepción, Chile. E-mail: evohringer@udec.cl

${ }^{c}$ Wilhelm-Ostwald-Institut für Physikalische und Theoretische Chemie, Universität Leipzig, Linné-Strasse 2, 04103 Leipzig, Germany

${ }^{d}$ Institut für Geowissenschaften, Universität Heidelberg, 69120 Heidelberg, Germany

${ }^{e}$ Institut für Raumfahrtsysteme, Universität Stuttgart, Pfaffenwaldring 29, 70569 Stuttgart, Germany

${ }^{f}$ University of Oulu, Astronomy and Space Physics, FI-90014 Oulu, Finland

${ }^{g}$ Leibniz-Institut für Oberflächenmodifizierung, Permoserstrasse 15, 04318 Leipzig, Germany

† Electronic supplementary information (ESI) available. See DOI: 10.1039/ c4cp05618c
}

in nature are in fact subject of debate and most of them are poorly understood.

For the sake of convenience we will address liquid and solid clusters, particles and fragments in the following altogether as droplets. We focus on three phenomena in which dispersed neutral water forms efficiently positively and negatively charged droplets in collisions. The common feature is the efficient dispersion of a larger molecular assembly into fast separating smaller subunits in a strong impact like a strong collision. The phenomenon is particularly puzzling because it works most efficiently in situations in which the total impact kinetic energy distributed over the particles and the kinetic energy per subunit (e.g., water molecules) is significantly lower than the molecular ionization potentials, so that charging cannot occur via ionization or the formation of a plasma. The energy is even insufficient to break a covalent bond.

The first phenomenon is related to neutral water clusters colliding with a wall at low kinetic energy, which has defied explanation up to the present. Vostrikov et al. ${ }^{11-13}$ and later Gebhard et al. ${ }^{1}$ observed in their experiments that molecular clusters colliding with a surface produce fragments that carry excess charges, even if the kinetic energy is well below any molecular ionization energy. This effect has been documented and there have been attempts to model this phenomenon, but a detailed understanding of the physical process is still lacking. Nevertheless, it has been used as a method of ionization in cluster mass spectrometry. ${ }^{1}$ 
Secondly, it has been a common belief that particle impact detectors based upon time-of-flight mass spectrometry of charged fragments work properly only if particles hit the impact plate with sufficient speed to form a plasma. ${ }^{14}$ This picture has been challenged recently by Postberg et al. ${ }^{6}$ Their measurements show that the composition of micron sized ice particles with low impact velocities can still be analyzed qualitatively and quantitatively via time-of-flight mass spectrometry, just from the charged fragments after destruction of the particles on the impact metal plate. These results imply that a fast dispersion of matter in a collision or impact will always produce charged fragments (see Fig. 1a). The mechanism, however, is unknown. A full account and quantitative explanation of the phenomenon - which is not plasma ionization or electron detachment from single molecules - has not been given yet.

Finally, charge generation in infrared laser assisted liquid beam, ${ }^{15-18}$ ice, ${ }^{19}$ or droplet ${ }^{20}$ dispersion mass spectrometry has been reported as a soft desorption and "ionization" technique, but it has not yet been understood on a quantitative nor at least qualitative basis. In this method a liquid beam containing charged particles $(\mathrm{NaCl})$ at various concentrations $\left(10^{-6}-10^{-1} \mathrm{~mol} \mathrm{~L}^{-1}\right)$ is dispersed by the irradiation of the infrared laser into droplets that are analyzed by mass spectrometry (see Fig. 1b). An interesting statistical model proposed by Dodd is frequently employed to explain charge states in mass spectrometry. ${ }^{21}$

Beyond these three application examples, other areas of our daily experience exist in which charge generation plays a role but our level of understanding appears to be even worse. For instance, in the field of cloud electrification and lightning formation, theoreticians have proposed a number of models, which are usually heavily debated and controversial (see ref. 2).

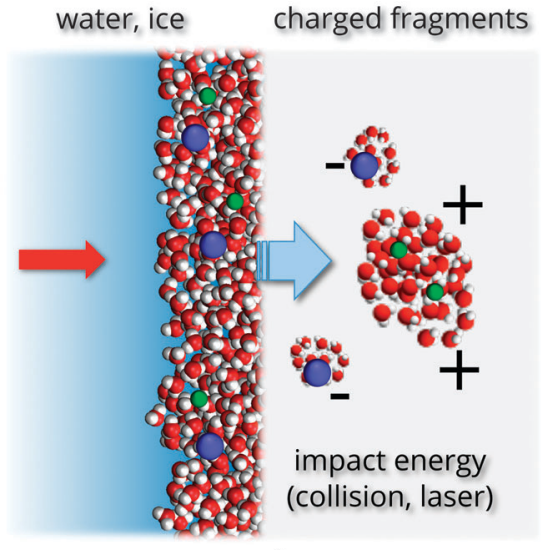

a)

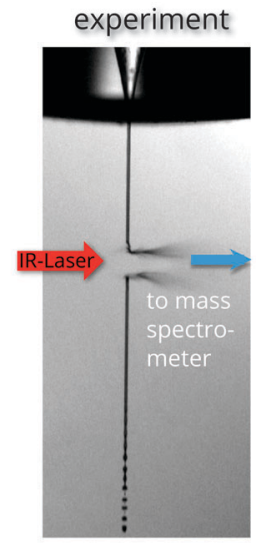

b)
Fig. 1 (a) Schematic picture of the phenomenon of charge separation and charged droplet formation after high-energy impact of water ice particles or water droplets containing charge carriers such as positively and negatively charged ions (e.g., from dissolved salts). The charge of a system is marked by - (negative excess charge) or + (positive excess charge). (b) A high-speed photograph of a micro water beam in vacuum (containing charge carriers) irradiated with an IR laser tuned to $2.8 \mu \mathrm{m}$. The micro beam is dispersed and the charged droplets are analyzed via a time-of-flight-mass spectrometer. For more details see the text and also the ESI $\uparrow$ of this article.
Despite the experimental evidence, theoretical approaches to describe charge separation in the dispersion of molecular aggregates or liquid water upon impacts are rare. Vostrikov et al. tried to explain their charged fragments in their early work on clusters colliding with a wall employing a molecular dynamics approach. They propose that the kinetic energy of clusters colliding with a surface concentrates in molecules of the compressed region resulting in what they called "polar dissociation of clustered $\mathrm{H}_{2} \mathrm{O}$ molecules". ${ }^{13}$ They concluded that for a given impact energy larger clusters form more efficiently ion pairs and that the surface may participate actively in the ion separation due to an "asymmetric ion neutralization" in a sub-picosecond time interval. ${ }^{13}$

Here, we propose a simple and intuitive but quantitative model that describes most of these phenomena either observed in nature or in lab experiments or even used as a working technology. The paper is organized as follows. First, we provide a general model for charge generation in high impact events (the Poisson model and Monte Carlo simulation). Then we compare predictions of the model with recent experiments from laser induced liquid desorption mass spectrometry. Finally, we show that the Poisson model contributes to the understanding of charge generation in cluster-impact mass spectrometry used in space based dust detectors at particle kinetic energies below the plasma threshold.

\section{Experimental and theoretical methods}

\section{a. Poisson model}

We start with a brief description of the Poisson model, which to the best of our knowledge - has not been considered nor discussed in the present context. This model is valid only at low salt concentrations, assuming that interactions between charge carriers can be neglected and that the separation process occurs fast relative to the mobility of the charge carriers. With these assumptions the distribution of the charged particles in the separated clusters is described by a Poisson distribution. As illustrated in Fig. 2 we assume a volume $V$ that contains uniformly distributed negative and positive non-interacting singly charged ions. Dividing the volume $V$ into $n$ parts yields a distribution of charged and neutral subspaces $V_{\mathrm{i}}$ (see Fig. 2) where the number of ions in these subspaces is Poisson distributed. Thus, the distribution of excess charges $q$ in these subspaces can be described by the sum of joint probabilities of the Poisson distributions of positive and negative ions.

To be specific, we assume a monovalent salt solution containing $\mathrm{Na}^{+}$and $\mathrm{Cl}^{-}$ions. The probability $P\left(N_{\mathrm{Na}}, k_{\mathrm{Na}}\right)$ for finding at a given instant in time $k_{\mathrm{Na}}$ sodium ions in a sub volume element is given by the Poisson distribution,

$$
P\left(N_{\mathrm{Na}}, k_{\mathrm{Na}}\right)=\frac{N_{\mathrm{Na}} k_{\mathrm{Na}}}{k_{\mathrm{Na}} !} \cdot \mathrm{e}^{-N_{\mathrm{Na}}},
$$

where $N_{\mathrm{Na}}$ is the expected number of sodium ions in the subspaces (calculated from a given concentration). A similar distribution holds for the chloride ions. This approach is similar in spirit to the one by Dodd. ${ }^{21}$

Hence, the total charge distribution $P(q)$ of these subspaces can be described by the sum of joint probabilities of the 


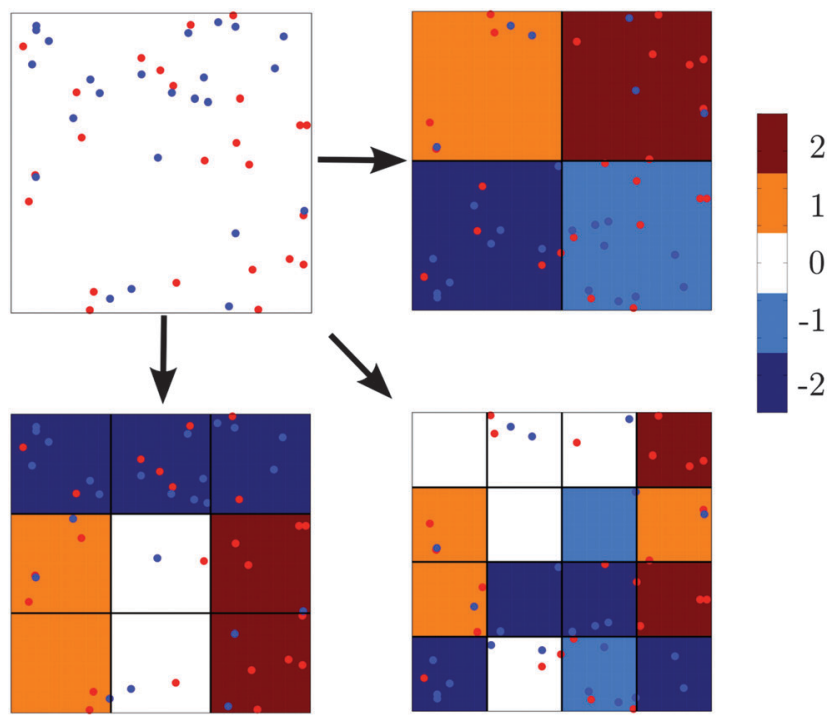

Fig. 2 Illustration of our simple "zeroth-order" statistical model for the phenomenon of charge separation in a water system (droplets or ice particles) containing positive and negative charge carriers (ions) that are dispersed or separated into sub-systems. The separation is assumed to be fast and the particles are assumed to be non-interacting which is typically the case for highly diluted systems at low ion concentrations. In this illustration we consider singly charged sodium ions labeled red and negatively charged chloride ions displayed as blue circles. The resulting subsystems of the initially neutral system indeed carry excess charge, depending on their statistical distribution. The resulting charge of the individual sub-systems is color-coded according to the scale bar (right hand side).

Poisson distributions of sodium and chloride ions, which yield an excess charge of $q$ in the corresponding subspace,

$$
P(q)=\sum_{k_{\mathrm{Na}}=0}^{\infty} \sum_{k_{\mathrm{Cl}}=0}^{\infty} P\left(N_{\mathrm{Na}}, k_{\mathrm{Na}}\right) \cdot P\left(N_{\mathrm{Cl}}, k_{\mathrm{Cl}}\right) \cdot \delta_{k_{\mathrm{Na}}, k_{\mathrm{Cl}}+q} .
$$

Approximating each of the two Poisson distributions in eqn (2) by a Gaussian distribution with mean $N$ and variance $N$, and replacing sums by integrals, yields, up to normalization, the useful approximation

$$
P(q) \propto \mathrm{e}^{\frac{-q^{2}}{4 N}} \cdot\left(1+\operatorname{erf}\left(\frac{2 N-q}{2 \sqrt{N}}\right)\right)
$$

or, for $q<2 \cdot \sqrt{N(N-1)}$,

$$
P(q) \propto \mathrm{e}^{\frac{-q^{2}}{4 N}}
$$

Accordingly, the width of the total charge distribution $P(q)$ is $\sqrt{2 N}, N$ being the expected number of ions in the corresponding subspace.

The total probability to obtain a charged droplet (a sub volume element) is $1-P(0)$. We will subsequently compare this probability with the experimentally obtained ion yield.

\section{b. Monte Carlo approach}

To account for the mutual Coulomb interaction between the ions, we performed Monte Carlo (MC) simulations of the ions in bulk water using a Metropolis scheme, ${ }^{22}$ and subsequently subdivided the MC volume into smaller cubic boxes to model instantaneous dispersion into droplets. From a fit of the Poisson model to the experimental ion yields at low concentrations $\left(c<0.01 \mathrm{~mol} \mathrm{~L}^{-1}\right)$, a droplet volume of $5600 \mathrm{~nm}^{3}$ was determined. Accordingly, a small cubic box of $17.8 \mathrm{~nm}$ side lengths was chosen.

For the MC simulations a cubic box with a side length of $17.8 \mathrm{~nm}$ plus six times the Debye length corresponding to the respective salt concentration was chosen to minimize surface effects. The number of ions was chosen from concentrations of $10^{-6} \mathrm{~mol} \mathrm{~L}^{-1}$ up to $0.1 \mathrm{~mol} \mathrm{~L}^{-1}$, to match the salt concentrations of the experiments described below. The electrostatic energy of the system was calculated from Coulomb's law. Water molecules were described implicitly using the relative permittivity of water $\varepsilon_{\mathrm{r}}=80.35$ for the electrostatic energy. MC steps in which the distance between two ions was smaller than the Lennard-Jones radius $(0.38 \mathrm{~nm}$, as defined in the Gromos 53a6 force field ${ }^{23}$ ) were rejected. All simulations have been performed at a temperature of $290 \mathrm{~K}$ and were equilibrated until the total energy $E$ of the system was converged to $\Delta E / E=10^{-7}$.

Finally, $10^{6}$ Monte Carlo steps have been computed to sample the ion distribution. For each concentration the charge distribution was derived counting the sodium and chloride ions in the center cubic volume. To achieve a converged charge distribution $125 \mathrm{MC}$ simulations have been performed for each salt concentration.

\section{c. Experimental}

A liquid beam with a diameter of about $14 \mu \mathrm{m}$ was formed by pumping doubly distilled water using a HPLC pump (see Fig. 1b) through a quartz nozzle in a vacuum in front of the mass spectrometer. Flow injection of the salt solutions was realized with a $100 \mathrm{ml}$ PEEK sample loop attached to an injection valve. The generated liquid jet consists of a continuous $\sim 3 \mathrm{~mm}$ long region, which disintegrates into droplets. The infrared laser beam $(2.9 \mu \mathrm{m}, 2.5 \mathrm{~mJ}$ per pulse) passed through a magnifying telescope and was focused by $\mathrm{a} \mathrm{CaF}_{2}$ lens through a $\mathrm{CaF}_{2}$ window onto the liquid target. A reflectron-Time-of-Flight mass spectrometer operating in the positive ion mode was employed to sample about 200 spectra per delay time $t(12-29 \mu \mathrm{s})$, which were averaged and summed up to obtain a total ion yield. ${ }^{18}$ $\mathrm{NaCl}$ p.a. was purchased from Merck. All solutions were freshly prepared with double distilled $\mathrm{H}_{2} \mathrm{O}$ before usage. More experimental details are given in ref. 15-18 and in ref. 6.

\section{Results and discussion}

To investigate fast charge separation and isolation in liquid water matrices after strong impacts experimentally and to compare it with theory, we pick a well-established and convenient mass spectrometry technique in our laboratory. ${ }^{15-18,24}$

The liquid water matrix, containing salt at various predefined bulk concentrations and therefore equal numbers of oppositely charged ions as charge carriers (i.e., $\mathrm{Na}^{+}$and $\mathrm{Cl}^{-}$ions), is dispersed without forming a plasma as illustrated in Fig. 1b 
(see also the high speed film material in the ESI $\dagger$ ). The irradiation of the liquid water jet in a thin seam of its surface region leads to a shock wave-induced anisotropic dispersion of the neutral water beam into charged micro and nano droplets. ${ }^{15}$ The charged droplets were detected using a charge sensitive detector (i.e., a multichannel plate, MCP) and characterized using a timeof-flight (TOF) mass spectrometer.

Such a laser experiment is readily able to simulate highenergy impacts, which are encountered in droplet collisions (both containing ions as charge carriers) or collisions of droplets with a wall. ${ }^{6}$ In order to study the dependence of droplet charging on the initial ion or charge carrier concentration, defined salt concentrations were added to bulk water, which results in aqueous salt solutions with concentrations between 0.1 and $10^{-6} \mathrm{~mol} \mathrm{~L}^{-1}$.

Mass spectra of $\mathrm{NaCl}$ aqueous solutions, dispersed using a high intensity pulsed IR-laser, are displayed in Fig. 3 for different salt concentrations. The detected droplets contain only one $\mathrm{Na}^{+}$-ion and up to five water molecules at concentrations lower than $10^{-3} \mathrm{~mol} \mathrm{\textrm {L } ^ { - 1 }}$ and display an increased intensity with bulk concentrations. Also a minor fraction of droplets charged by one proton or by hydroxide ions (in combination with two sodium ions) due to the self-ionization of water is observed. For higher concentrations droplets might also contain up to three or more ions. We emphasize here that in the experiment nearly exclusively singly charged water droplets are observed, which has been reported and well documented in the past ${ }^{15}$ and which is verified also in this work. In the experiments the intensities corresponding to $\mathrm{Na}^{+}$-ions at all masses are summed up for the different ion concentrations (providing a measure for the ion yield) and compared with theoretical predictions outlined above (see Fig. 4).
Fig. 4 compares the measured ion yield (blue circles) with the predictions of both the simple Poisson model (black line, no interionic interactions) and the MC-model (with interionic interactions, red symbols). Here, we have assumed that the average size of the droplets does not depend on the salt concentration. This size, $5.6 \pm 3.2 \times 10^{3} \mathrm{~nm}^{3}$, was determined from a weighted (with experimental error) fit of the Poisson model to the experimental ion yields below $10^{-2} \mathrm{~mol} \mathrm{~L}^{-1}$ salt concentration; this is in fact the only fit parameter of the theory and yields the expected number of ions $N$ for each concentration. On average, droplets of this size contain less than one ion at salt concentrations lower than $3 \times 10^{-4} \mathrm{~mol} \mathrm{~L}^{-1}$, which agrees with the observed droplet mass spectra in Fig. 3. It should be emphasized that the spectra are not modeled per se (line by line) but the total charge from the integrated line spectrum. We note that in our treatment we assume an average droplet size and neglect the actual width of the unknown size distribution. This simplification will add to the deviation we see between our Poisson model and the experimental data contributing to the observed error in the droplet volume. In the laboratory spectra from Fig. 3 we see clusters that are significantly smaller than $5.6 \pm 3.2 \times 10^{3} \mathrm{~nm}^{3}$ obtained from fits to the Poisson model. This reduction in size is likely caused by the heat induced by the $7 \mathrm{~ns}$ laser pulse, leading to a substantial evaporation of the cluster on the way to the detector. This evaporation, however, leaves the ion yield unaffected at low concentrations.

As can be seen in Fig. 4, the Poisson model describes the charge distribution after strong laser dispersion at low salt concentrations up to $10^{-3}$ mol $\mathrm{L}^{-1}$ indeed very well. With increasing salt concentration the interionic interactions become more relevant, and above $10^{-3} \mathrm{~mol} \mathrm{~L}^{-1}$ these interactions markedly narrow the charge probability distributions as

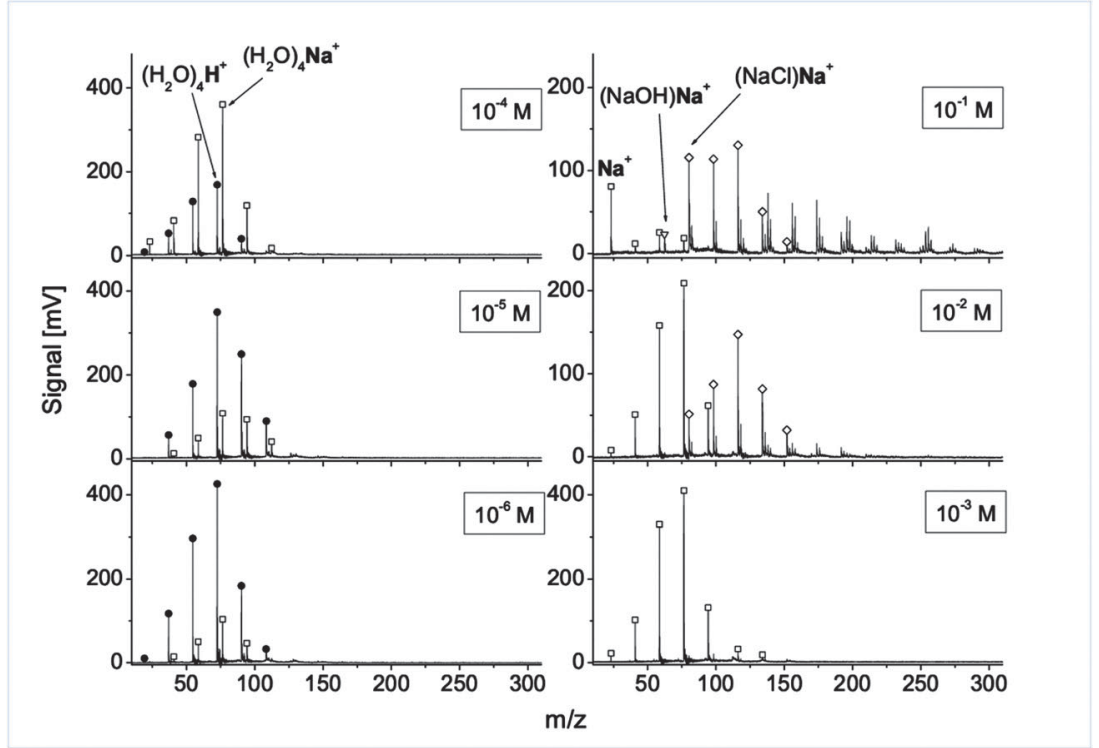

Fig. 3 Time of flight mass spectra of laser dispersed aqueous sodium chloride solution at six different concentrations, between $10^{-1}$ mol $\mathrm{L}^{-1}$ and $10^{-6} \mathrm{~mol} \mathrm{~L}{ }^{-1}$. Circles label $\left(\mathrm{H}_{2} \mathrm{O}\right)_{n} \mathrm{H}^{+}$water aggregates (clusters), open squares $\left(\mathrm{H}_{2} \mathrm{O}\right)_{n} \mathrm{Na}^{+}$clusters, open diamonds label characteristic mixed $\left(\mathrm{H}_{2} \mathrm{O}\right)_{n}(\mathrm{NaCl})_{n} \mathrm{Na}^{+}$aggregates, and open triangles denote $(\mathrm{NaOH}) \mathrm{Na}^{+}$clusters. 


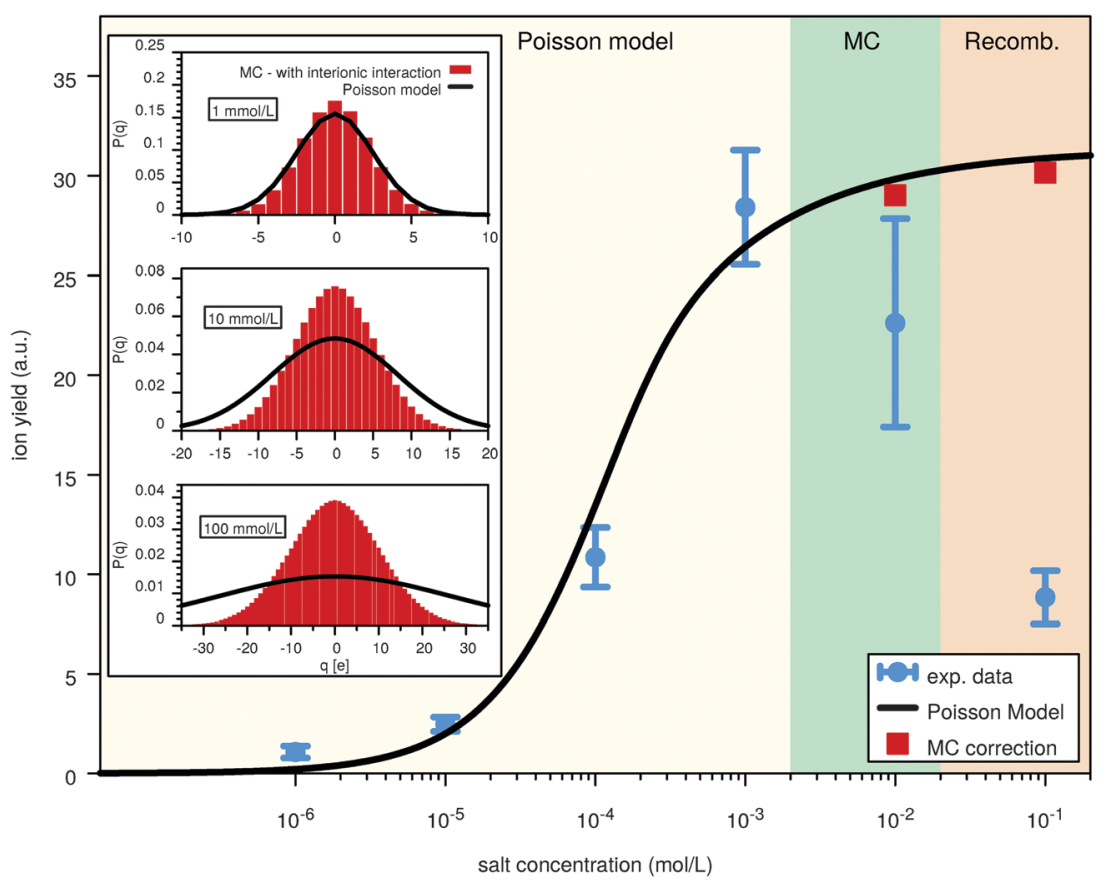

Fig. 4 Comparison of experiment and theory. The blue filled circles represent the measured total ion yield as a function of salt concentration, i.e., the sum of all mass spectrum intensities obtained from droplets containing sodium (Fig. 3). The black line shows the prediction of our Poisson model, with the droplet volume as the only adjustable parameter. The red boxes represent ion yields predicted by our Monte Carlo simulations. Three concentration regions discussed in the text are indicated by background colors. Inset: total charge probability distribution for three selected concentrations, assuming the same droplet size as in the main figure.

shown in the inset of Fig. 4 (for lower concentrations the match between the Poisson model and MC simulations is complete, see the ESI $\dagger$ ). The respective correction obtained from the MC simulation for concentrations of $10^{-2} \mathrm{~mol} \mathrm{~L}^{-1}$ and $10^{-1} \mathrm{~mol} \mathrm{~L}^{-1}$ is shown as a red box. As a consequence, the correction decreases the ion yields, a tendency that is observed in the experiment, albeit at a much larger extent.

Finally, at very high salt concentrations the effect of overall strong charge recombination becomes significant, and the ion yield drops significantly such that the simple static model breaks down. We assume that for concentration above $10^{-2} \mathrm{~mol} \mathrm{~L}^{-1}$ the assumption of an instantaneous charge separation becomes questionable: for very high concentrations, already the short diffusion distances of the ions during the actual dispersion process entail large changes in the total charge. The consideration of this complex dynamics would require full atomistic molecular dynamics simulations, which are however beyond the scope of this work.

Overall, for low and intermediate salt concentrations below $0.01 \mathrm{~mol} \mathrm{~L}^{-1}$, our Poisson model and the MC simulation predict the charge generation after (fast) dispersion of neutral aqueous systems ( $\mathrm{NaCl}$ salt solutions) quite well. It should be noted that the internal energies of the water beam are well below the ionization limit, such that the charge 'generation' originates from dispersion only and not from ionization.

It is quite surprising that the simple Poisson model, which rests on very few and general assumptions, obviously describes the process so well. This finding suggests that in fact a wide variety of charging processes in nature - ranging from charged oil droplets ${ }^{21}$ to charged water aggregates in mass spectrometry experiments - might be explained by a similar process as long as the interactions between the charge carriers are small. ${ }^{15,17}$ The charge probability in the experiments above is indeed very well described at low salt concentrations, i.e. in the regime where the interaction of the ions is negligible, and the average number of charge carriers (ions) is less than one per droplet.

At higher salt concentrations, the interionic interactions become relevant; they are therefore considered within the MC simulations for concentrations higher than $10^{-3} \mathrm{~mol} \mathrm{~L}^{-1}$. Due to these interactions, the radial distribution function of oppositely charged ion pairs is shifted to smaller distances, which implies a somewhat reduced overall ion yield after the dispersion process, in agreement with the experiment (see Fig. 4).

Experiments at even higher salt concentration (see Fig. 4) show a dramatic decrease in the ion yield. This is in contrast to both the simple statistical Poisson model that predicts a quite broad plateau as well as the MC model, which predicts only a slight decrease. We conclude that there must be a very efficient dynamic mechanism, which markedly reduces the probability of charged fragments, further than the simple equilibrium (MC) model. We assume that at these concentrations ioncluster collisions with ion uptake and intra-cluster recombination are efficient enough to explain the reduced ion yields in the mass spectrometry experiments.

Does our model have predictive power beyond the observed agreement? As a matter of fact, from our theoretical approach, the production of charged fragments even below any molecular ionization energy described in the paper of Gebhard et al. can 
now even be understood on a qualitative basis. ${ }^{1}$ Obviously, charge separation and charging of fragments arise naturally in impacts of clusters. In light of the present work, the calculations of Vostrikov et al. on charge separation in the compressed region of a cluster do not seem to capture the main effect. ${ }^{13}$ Instead, the present work naturally explains experimental results that have been highlighted and highly appreciated by the scientific community, but which were yet difficult to explain and rationalize in a qualitative and quantitative fashion via rigorous theory in comparison with experiment. Additionally, our Poisson model also agrees with the previously observed Gaussian distribution of charges in experiments with dispersed oil droplets. ${ }^{21}$

It is of course tempting to speculate that our model is also relevant for charge separation in thunderstorms, ultimately responsible for lightning. Although the present work does provide new insights into charge separation and generation in droplets and particles of water after high-energy impacts, the overall mechanism of atmospheric charging and lightning formation is very complex and certainly involves more than just a single mechanism. To establish such relevance for these complex phenomena which have resisted a detailed understanding for decades ${ }^{2,9,25,26}$ is, as exciting as it may be, certainly beyond the scope of this work.

Another application of the charge generation and separation concept highlighted here is mass spectrometry from impact ionization of small dust particles (typical size is on the order of $\mu \mathrm{m}$ or tens of $\mu \mathrm{m}$ ). This method has originally been designed for impact speeds in the order of $10 \mathrm{~km} \mathrm{~s}^{-1}$ or higher. ${ }^{14}$ In this speed regime energy densities are in general high enough to directly ionize portions of the dust particle upon impact in a plasma plume. However, laboratory experiments using a dust accelerator indicated that there is abundant formation of charged clusters already at smaller impact speeds, especially for elements and molecules with low ionization energies. ${ }^{27,28}$ This effect was directly seen in ice particle measurements performed by the dust detector onboard the Cassini spacecraft at Saturn. Previously, we have shown that the recorded spectra at smaller impact speeds can be simulated in our laboratory with the same liquid beam desorption mass spectrometry described above (ref. 6).

This suggests that ice particles in space can be analyzed via the dispersion mechanism described above, even without plasma formation. It allows new applications for space missions like compositional measurements of dust from an orbit around a planetary moon. ${ }^{29,30}$ Namely, the impact speeds of ejecta lifted or emitted from the moon's surface onto the dust sensor are typically on the order of 1-2 $\mathrm{km} \mathrm{s}^{-1}$ (e.g. for an orbit of about $100 \mathrm{~km}$ altitude around Jupiter's moons Ganymede or Europa). Such measurements can provide key chemical constraints for revealing the satellite's surface composition. Dust particles are small samples from the solid surface of a satellite such as Earth's moon or the Galilean satellites. In the absence of a lander, simply analyzing the ejected dust particles in the vicinity of the satellite and its exosphere can carry out a composition analysis of a satellite's surface.

\section{Summary and conclusions}

In conclusion, we have found that dispersion of liquids, droplets or ice particles containing charge carriers produces charged fragments (aggregates and droplets) if they are dispersed fast in a strong impact. Although the total impact energy distributed over each particle is below its ionization potential, charge generation is accomplished by breaking up the liquid or solid matrix mechanically into charged fragments.

The charge state distributions can be predicted in the low and intermediate concentration range and are described by a simple statistical Poisson model. At higher salt concentrations, the interionic interactions become important, which are described (at least in part) by Monte Carlo simulations. These results are in good agreement with the experimentally observed fractions of charged droplets after infrared laser assisted liquid phase dispersion mass spectrometry.

Our model of charge separation and generation appears to be of general nature and should be able to explain a variety of phenomena in science and nature. In particular, the model may also be applied to the characterization of dust particles collected in the exosphere of satellites such as the Jovian moon Europa, providing information on their surface composition.

\section{Acknowledgements}

The authors thank the Fonds der Chemischen Industrie, the Volkswagenstiftung, and in particular the Deutsche Forschungsgemeinschaft DFG for financial support (Project AB63/12-1, Projekt AB 63/9-1, Projekt PO 1015/2-1). E.V.M. also thanks Fondecyt No. 11121179 and Grant ICM No. 120082. F.W. and H.G. thank for funding by the DFG, SFB 755. Interesting discussions with U. Buck and S. Kempf are gratefully acknowledged.

\section{References}

1 C. R. Gebhard, H. Schroeder and K.-L. Kompa, Nature, 1999, 400, 544.

2 P. Jungwirth, D. Rosenfeld and V. Buch, Atmos. Res., 2004, 76, 190.

3 P. Lenard, Ann. Phys., 1892, 46, 584.

4 J. Mason and N. Mason, Eur. J. Phys., 2003, 24, 99.

5 T. Pähtz, H. J. Herrmann and T. Shinbrot, Nat. Phys., 2010, 6, 364 .

6 F. Postberg, S. Kempf, J. Schmidt, N. Brilliantov, A. Beinsen, B. Abel, U. Buck and R. Srama, Nature, 2009, 459, 1098.

7 D. C. Blanchard, Progress in Oceanography, Pergamon, Elmsford, 1963, vol. 1.

8 C. Saunders, Planetary Atmospheric Electricity, Springer, 2008.

9 T. Takahashi, J. Atmos. Sci., 1978, 35, 1536.

10 A. M. Duffin and R. J. Saykally, J. Phys. Chem. C, 2008, 112, 17018.

11 A. A. Vostrikov and D. Y. Dubov, Z. Phys. D: At., Mol. Clusters, 1991, 20, 61. 
12 A. A. Vostrikov, D. Y. Dubov and M. R. Predtechenskiy, Chem. Phys. Lett., 1987, 139, 124.

13 A. A. Vostrikov, A. M. Zadorozhny, D. Y. Dubov, G. Witt, I. V. Kazakova, O. A. Bragin, V. G. Kazakov, V. N. Kikhtenko and A. A. Tyutin, Z. Phys. D: At., Mol. Clusters, 1997, 40, 542.

14 R. Srama, Space Sci. Rev., 2004, 114, 465.

15 A. Charvat and B. Abel, Phys. Chem. Chem. Phys., 2007, 9, 3335.

16 A. Charvat, A. Bogehold and B. Abel, Aust. J. Chem., 2006, 59, 81.

17 A. Charvat, E. Lugovoj, M. Faubel and B. Abel, Eur. Phys. J. D, 2002, 20, 573.

18 A. Charvat, E. Lugovoj, M. Faubel and B. Abel, Rev. Sci. Instrum., 2004, 75, 1209.

19 S. Berkenkamp, M. Karas and F. Hillenkamp, Proc. Natl. Acad. Sci. U. S. A., 1996, 93, 7003.

20 E. Rapp, A. Charvat, A. Beinsen, U. Plessmann, U. Reichl, A. Seidel-Morgenstern, H. Urlaub and B. Abel, Anal. Chem., 2009, 81, 443.

21 E. E. Dodd, J. Appl. Phys., 1953, 24, 73.

22 N. Metropolis, A. W. Rosenbluth, M. N. Rosenbluth, A. H. Teller and E. Teller, J. Chem. Phys., 1953, 21, 1087.
23 C. Oostenbrink, A. Villa, A. E. Mark and W. Van Gunsteren, J. Comput. Chem., 2004, 25, 1656-1676.

24 O. Link, E. Vohringer-Martinez, E. Lugovoj, Y. X. Liu, K. Siefermann, M. Faubel, H. Grubmuller, R. B. Gerber, Y. Miller and B. Abel, Faraday Discuss., 2009, 141, 67.

25 E. E. Avila, G. M. Caranti, N. E. Casteellano and C. P. R. Saunders, J. Geophys. Res., 1998, 103, 8985.

26 E. E. Avila, G. S. Longo and R. E. Bürgesser, Atmos. Res., 2003, 69, 99.

27 F. Postberg, J. Schmidt, J. Hillier, S. Kempf and R. Srama, Nature, 2011, 474, 620-622.

28 J. K. Hillier, F. Postberg, S. Sestak, R. Srama, S. Kempf, M. Trieloff, Z. Sternovsky and S. F. Green, J. Geophys. Res., 2012, 117, 2156-2202.

29 F. Postberg, E. Gruen, M. Horanyi, S. Kempf, H. Krueger, J. Schmidt, F. Spahn, R. Srama, Z. Sternovsky and M. Trieloff, Planet. Space Sci., 2011, 59, 1815-1825.

30 S. Kempf, R. Srama, E. Gruen, A. Mocker, F. Postberg, J. Hillier, M. Horanyi, Z. Sternovsky, B. Abel, A. Beinsen, R. Thissen, J. Schmidt, F. Spahn and N. Altobelli, Planet. Space Sci., 2012, 65, 10-20. 